\title{
Design and Feasibility of Thermally Tuned Thin Film Filters for NG-PON2 System Applications
}

\author{
Zoran Vujicic $^{\mathrm{a},{ }^{*}}$, Berta Neto ${ }^{\mathrm{b}}$, Ricardo Bastos ${ }^{\mathrm{b}}$, Antonio Teixeira ${ }^{\mathrm{b}}$ and Jonathan Rodriguez ${ }^{\mathrm{a}, \mathrm{b}}$. \\ a Department of Electronics, Telecommunications and Informatics, University of Aveiro, Campus Universitario \\ de Santiago, 3810-193, Aveiro, Portugal. \\ bInstituto de Telecomunicações, Campus Universitário de Santiago, 3810-193 Aveiro, Portugal
}

\begin{abstract}
We investigate the feasibility of low-cost, compact optical filtering for NG-PON2 based on thermally-tuned thin film filters. By considering several contrasting designs in a comparative assessment of their optical performance, complexity, tuning time and power efficiency, we identify relevant technical tradeoffs towards their optimized and low-cost application in NG-PON2 systems.
\end{abstract}

Index Terms - Next Generation Passive Optical Networks (NG-PON), Wavelength Division Multiplexing (WDM), Thin Film Filters (TFF), Thermo-electric Control (TEC).

\section{INTRODUCTION}

$\mathrm{T}$ OWARDS meeting the ever growing demand for bandwidth and service versatility in next generation communication networks, the full service access network (FSAN) group has published the Next Generation Passive Optical Networks (NGPON2) standards and, more recently, the second amendment on Physical Media Dependent (PMD) layer specification [1]. The roadmap for PON beyond NG-PON2 foresees a capacity growth leveraged by market segmentation by subscriber type, pay-asyou-grow transition paradigm and the optical bandwidth demand of the mobile fronthaul [2]. Thus, per channel line rates are expected to increase from the legacy $10 \mathrm{~Gb} / \mathrm{s}$ to $25 \mathrm{~Gb} / \mathrm{s}$ in both transmission directions [3], leading to new technical challenges in terms of reduced optical power budget and wavelength stability compliance with WDM. According to a report from Ovum organization, the average selling price of an NG-PON2 optical network unit (ONU) is predicted to be more than 20 times that of a GPON ONU [4]. Therefore, it is essential to drive down the cost of the NG-PON2 transceivers, namely by using affordable and effective filtering solutions, compliant with the current standards but flexible enough to enable upgrades in future PON transceivers. As standardization requirements evolve along with the cost/quality of state-of-the-art system components resulting in reduced tolerances towards previously standardised criteria, the relevance of cost/complexity, stability and tuning time performance of optical filtering is ever more pronounced [5].

Considering that Photonic Integrated Circuits (PICs) are also expected to support the implementation of high performance transceivers for both optical line terminals (OLTs) and ONUs, all contributions to reduce the photonic process costs must be taken into account. Packaging is responsible for nearly $30 \%$ of the overall cost of manufacturing of PIC modules [6], whereas the optical coupling alignment with the optical fiber is especially complex since (i) end-facet coupling requires sub-micron alignment precision and (ii) grating couplers limit the spectral bandwidth and introduce additional complexity to the module design [7]. Zhang et al. proposed a solution that facilitates the coupling issues while adding optical functionalities [8]. In this approach, a Thin Film Filter (TFF) is used in the polymer platform for both transmission directions to separate the C/L bands avoiding the use of high footprint filtering elements such as Arrayed Waveguide Gratings (AWGs) in the InP PIC, and consequently reducing the solution cost [8]. Thus, TFFs offer efficient filtering characteristics at a very small footprint.

Typically, both tuneable TFFs and AWGs meet the requirements for colourless coexistence-type PONs [9,10]. Angle-tuned TFFs, driven by microelectromechanical systems (MEMS) of micro-mirrors, use low thermo-optic coefficient materials towards increased wavelength stability [9] and achieve wavelength tuning by varying the incident angle. Angle tuned TFFs for NG-PON2 ONU receivers were proposed by Scholtz et al [11] for 8 channels with $100 \mathrm{GHz}$ spacing. The reported scenarios considered both greenfield NG-PON2 and coexistence with other PON technologies. However, the implementation of reported angles would require a tight control, along with high tuning angles in coexistence scenarios, which may pose a feasibility challenge. Namely, electrostatically actuated MEMS micro-mirrors are suitable for precise positioning and accurate beam steering, but the resolution is $0.2^{\circ}$ and the scan range is typically limited to $20^{\circ}$ [12]. Although many efforts in the past decade improved the integration of MEMS with the transceiver PIC, the footprint of such devices is still considerable $[13,14]$. Cost and complexity aspects associated with large-volume manufacturing of mechanically tuned TFFs is another potentially deleterious factor. Additionally, polarization dependent loss (PDL) is typically an issue in angle tuned TFFs [15], and may significantly affect the overall system

*Corresponding author

E-mail addresses: zvujicic@av.it.pt (Z.Vujicic),bneto@av.it.pt (B. Neto), ricardo.bastos@ua.pt (R. Bastos), teixeira@ua.pt (A. Teixeira), jonathan@av.it.pt (J. Rodriguez). 
performance.

Thermo-optical tunability is an alternative approach, however, the following requirements ought to be accounted for: i) spectral tuning of at least $300 \mathrm{GHz}$ (for 4 channels NG-PON2 use case); ii) tuning time compliant with NG-PON2 standards for thermally actuated devices - class 3 (from $25 \mathrm{~ms}$ to $1 \mathrm{~s}$ ); and iii) targeting energy efficiency. Typically, thermally actuated devices underperform in terms of energy efficiency when compared with electrostatically driven ones. Thermally tuned AWGs in the silicon-based platform using a TiN heater were recently proposed for WDM applications by Yang et al [16], achieving 600 $\mathrm{GHz}$ of tuning range by applying a temperature increment of $53^{\circ} \mathrm{C}$ compared to the room temperature $\left(25^{\circ} \mathrm{C}\right)$. However, the required bias power for wavelength shifts beyond $2.5 \mathrm{~nm}(300 \mathrm{GHz})$ exceeds $0.5 \mathrm{~W}$. Furthermore, AWGs are typically larger sized when compared to TFFs and have higher insertion losses and lower isolation when flat top passband shape is pursued [17]. Nonetheless, isolation and insertion loss may vary with temperature in both cases, where TFFs typically exhibit a stable behavior in a wider temperature range in practical implementations [18]. However, to enable requirement (i) for TFF design, materials that present high thermo-optic coefficients (i.e. amorphous semiconductors) are to be considered. By using such materials in their design, Domash et al. have previously proposed to subvert the typical thin-film stack material choice logic of aiming for low thermal variability, [10,19]. Amorphous silicon (a-Si) and silicon nitride (a-SiN) exhibit excellent transparency in the near infrared (NIR) and high refractive index, but it is their high thermo-optic coefficient with stable behavior within the NIR spectral region that enables tuning applications [20-22]. Furthermore, these also benefit from the high integrability with silicon photonics circuits. However, such solution still requires a large temperature increment in tuning scenarios [10]. In order to overcome these issues, identifying materials with suitable properties for optimized TFF design is of great importance. Moreover, the field remains largely unexplored for system implications of TFF design in NG-PON2 scenario and beyond, where it is essential to address common issues found in applications of thermally tuned devices, particularly in L-band operation, which include typically low tuning speed, demanding wavelength stabilization and high power consumption [22].

Hereby, we propose TFF designs optimization methods for NG-PON2 system scenario based on thermal tuning, and extensively identify their system level advantages and pitfalls compared to angle tuning optimized designs. We showcase unique design formulas for ONU applications operating in L-band with $100 \mathrm{GHz}$ of channel spacing and, to the best of our knowledge, beyond state of the art for transmittance in-band flatness, sharpness and stability for thermally tuned TFFs. In pursuit of higher energy efficiency compared to the state of the art designs, we seek improvements in thermal tunability and impedances by basing our design on germanium in contrast to amorphous silicon [22, 24, 25]. Complexity versus crosstalk isolation performance tradeoff is addressed for the first time, and benchmarked against that of typical AWG and comparable TFF designs.

This paper is structured as follows. Section 2 describes the TFF design rationale and optimization methodology, while addressing the complexity versus performance tradeoff. Tuning stability and range are investigated in Section 3 and compared to the angle tuning scenario. Systemic analysis of filter performance in two use cases of NG-PON2 scenario is presented in Section 4, while Section 5 outlines the design repercussions on tuning time and power consumption. Finally, main conclusions are summarized in Section 5.

\section{Design AND TRAnSmitTAnce Optimization}

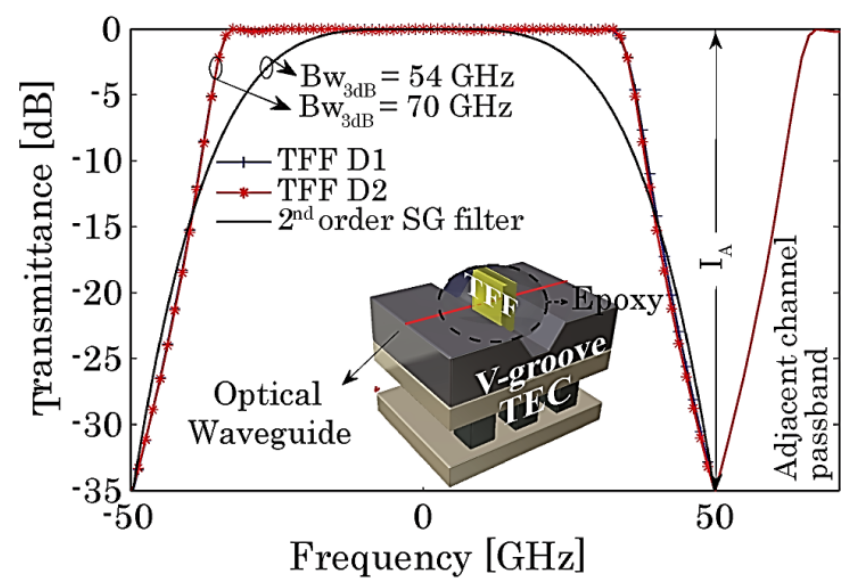

Fig. 1. D1 and D2 transmittance compared to that of $2^{\text {nd }}$ order super-Gaussian (SG) considering $100 \mathrm{GHz}$ channel spacing. The inset shows hybrid photonic assembly, integrating TFF with optical waveguides and TEC. Adjacent channel isolation $\mathrm{I}_{\mathrm{A}}$ presented is $35 \mathrm{~dB}$.

The methodology for theoretical considerations in this work is based on the transfer matrix formalism, as a form of the steadystate solution to Maxwell's equations, subject to the boundary conditions imposed by a multilayer stack [19]. Transfer matrix formalism is coupled with genetic algorithm (GA) based optimization, using filter flatness within the band-pass region as the fitness criterion. In our approach, the proper choice of the GA operators and initial population size enabled an efficient 
convergence to optimal parameters while avoiding premature convergence. Thus, the elected operators are stochastic uniform for selection, scattered for crossover and uniform for mutation. A population size of 200 was considered optimal, leading to convergence typically after 50 generations. Tuning time and power consumption investigation in the latter stages were carried out using ANSYS ${ }^{\circledR}$ software, adopting the design geometry depicted in Fig.1. The adopted design geometry with respect to hybrid photonic integration allows vertically inserted TFF assembly on a chip V-groove, enabling compact integration with thermo-electric controller (TEC), as depicted in the inset of Fig.1. Additionally, epoxy-based optical adhesive is adopted as incident medium (Inc.), towards providing thermal insulation and reducing free-space propagation-induced optical loss. $50 \mu \mathrm{m}$ thick silicon $(\mathrm{Si})$ wafer is considered for the substrate $(\mathrm{Sub}$.) due to its excellent thermal conductivity.

Following mentioned methodology, two unique multi-cavity designs (D1, D2), with GA optimized admittance matching for $35 \mathrm{~dB}$ rejection ratio over $100 \mathrm{GHz}$ channel spacing in L-band [1], are derived:

Design 1 (D1):

$$
\begin{array}{ll}
\text { Inc. } & \mid 12.187 \mathrm{H} 11.497 \mathrm{~L}(\mathrm{HL})^{3} \mathrm{H}(\mathrm{HL})^{8} \\
& \\
\mathrm{H}\left(3 \mathrm{H} 2 \mathrm{~L} 3 \mathrm{H}(\mathrm{HL})^{8} 4 \mathrm{H} 2 \mathrm{~L} 3 \mathrm{H}\right)^{\mathrm{R}} & \\
(\mathrm{HL})^{8} \mathrm{H}(\mathrm{HL})^{3} \mathrm{H} 21.008 \mathrm{~L} 23.844 \mathrm{H} & \text { I Sub. }
\end{array}
$$

Design 2 (D2):

$$
\begin{array}{l|l}
\text { Inc. } & \text { 5.798H 4.604L (HL) }{ }^{2} \mathrm{H}(\mathrm{HL})^{6} \mathrm{H} \\
& \left(16 \mathrm{H}(\mathrm{HL})^{6} 17 \mathrm{H}\right)^{\mathrm{R}}(\mathrm{HL})^{6} \mathrm{H}(\mathrm{HL})^{2} \mathrm{H} \\
& 4.037 \mathrm{~L} 2.025 \mathrm{H} 2.837 \mathrm{~L} 3.824 \mathrm{H}
\end{array}
$$

\begin{tabular}{|c|c|c|c|c|c|}
\hline Layer $^{\text {Design }}$ & Incidence $^{1,2}$ & Substrate $^{1,2}$ & $\mathbf{H}^{1}$ & $\mathbf{H}^{2}$ & $\mathbf{L}^{1,2}$ \\
\hline Type & Epoxy $^{[11]}$ & $\mathbf{S i} \mathbf{i}^{[12,13]}$ & a-Si-H ${ }^{[6],[14]}$ & a-Ge ${ }^{[10],[12],[15]}$ & $\mathbf{a}-\mathbf{S i}_{\mathbf{x}} \mathbf{N}_{\mathbf{y}}-\mathbf{H}^{[6],[16]}$ \\
\hline$n$ & 1.54 & 3.47 & 3.71 & 4.2 & 1.77 \\
\hline$d n / d T\left[\square C^{-1}\right]$ & $-1.7 \cdot 10^{-4}$ & $6.7 \cdot 10^{-5}$ & $2.3 \cdot 10^{-4}$ & $6 \cdot 10^{-4}$ & $4.6 \cdot 10^{-5}$ \\
\hline$k[\mathbf{W} / \mathbf{m K}]$ & 0.2 & 149 & 1.1 & 5.68 & 3.25 \\
\hline
\end{tabular}

The design rationale for these may be summarized as follows: i) achieving superb flatness and sharpness for the challenging target bandwidth with acceptable layer count compared to state of the art solutions; ii) minimizing angular dependency and subsequent sensitivity to oblique incidence; and iii) boosting temperature tunability and thus minimizing transmittance degradation occurring away from the optimal wavelength. The latter is also expected to yield reduced power consumption.

Table 1: Layer and TFF design properties.

These aims were pursued by using the following design methodology. Providing sufficient contrast between high $(\mathrm{H})$ and low (L) layer refractive indices is essential for narrow bandwidth TFF design and high maximum spectral isolation. In order to monitor the effect of filter sharpness in our analysis, we highlight the main period repetition parameter $\mathrm{R}$ as the dominant factor. Outside the main period, additional stacks are added on each end side for substrate and incidence matching. Namely, deviation of physical thickness from quarter-wave (QW) value of the compensation layers added to the multi-cavity TFF, on the incident and substrate interfaces respectively, plays a crucial role in filter flatness [19]. The latter were derived using GA optimization for optimal matching within the tuning range of $300 \mathrm{GHz}$. Next, reduced sensitivity to oblique incidence due to possible misalignments within the PIC is assured by adopting designs with high refractive index spacer. It is important to note that, as the required temperature increment $\Delta \mathrm{T}$ grows for less thermally efficient solutions, so does the need to balance the spacer with $\mathrm{L}$ layers to maintain the transmittance shape - which in turn boosts angle misalignment sensitivity. Such is the case with D1 compared to D2. Additionally, deviation from normal incidence leads to the transmission peak shift towards shorter wavelengths and may introduce considerable PDL due to the central wavelength separation [15]. These design aspects will be addressed in the following section, as they showcase the importance of scenario specific trade-off in TFF material selection. Finally, improvement of transmittance stability away from the optimal quarter-wave is sought through the utilization of high thermooptic coefficient materials, with the stringent condition of being highly compatible with photonic integration technology. Table 1 outlines the materials used for the layers comprising each design, including their respective refractive index $n$, thermo-optic coefficient $d n / d T$, and conductivity $k$. The relevant parameters from Table 1, available for C-band, were adjusted for their dependency on wavelength using functions based on state of the art experimental data [20-22], [24-28].

The resulting transmittance of proposed designs for $\mathrm{R}=6$, showcasing $100 \mathrm{GHz}$ bandwidth response at $35 \mathrm{~dB}$ of adjacent channel isolation $\mathrm{I}_{\mathrm{A}}$ are presented in Fig. 1 and compared to the flat-top $2^{\text {nd }}$ order super Gaussian (SG) filter response (i.e. ideal AWG). We hereby define the adjacent channel isolation at the limit of its transmission bandwidth for $100 \mathrm{GHz}$ channel spacing grid, denoted $I_{A}$. Upon design optimization, both final TFF designs obtained showcased the $3 \mathrm{~dB}$-to-35dB bandwidth isolation 
ratio of 0.7 , indicating excellent sharpness. Square optical filter (OFIL) response is essential in telecom applications, especially towards increased spectral efficiency and technology convergence scenarios requiring optimized millimeter-wave transmission [29]. Moreover, both filters retain flatness across the tuning range used in the optimization procedure, maximum ripple being $0.26 \mathrm{~dB}$, as demonstrated in Fig.2. GA optimization results presented in Fig.2 further indicate that, due to the higher ratio between $\mathrm{H}$ and $\mathrm{L}$ indices in $\mathrm{D} 2$, the latter required a compensation stack with 6 additional layers, as opposed to 4 required for the specified flatness in D1. Thus, it is worth noting that a further increase in refractive index contrast may lead to increased design complexity.

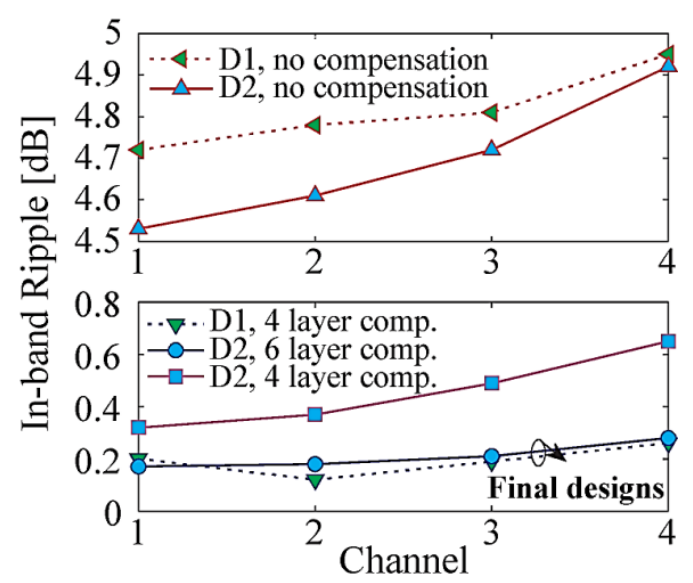

Fig. 2. D1 and D2 transmittance in-band ripple over 4-channel tuning range without (upper) and with matching compensation layers (lower), and the elected final designs based on low ripple performance.

For $I_{A}=35 \mathrm{~dB}$, both designs rely on over 200 layers, which may be offset in future applications relying on spectrally efficient modulation formats in accordance with their spectral occupation and optimal filtering bandwidth [5], by reducing filter sharpness (R parameter). Fig.3 shows the adjacent channel isolation, $\mathrm{I}_{\mathrm{A}}$, for $100 \mathrm{GHz}$ spacing versus the resulting layer count, obtained by reducing stack repetitions towards lower filter sharpness. Consequently, both designs retain flatness for reduced $\mathrm{I}_{\mathrm{A}}$, and thus may be optimized for layer count providing scenario specific performance versus complexity trade-off.

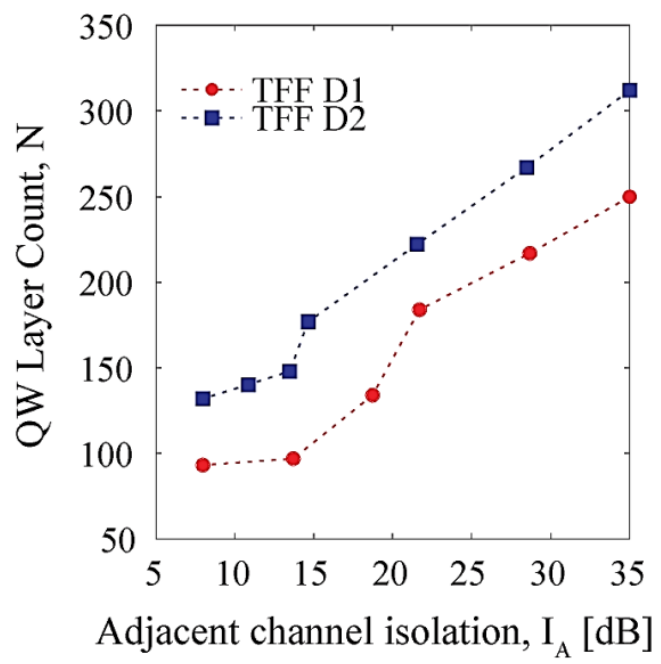

Fig. 3. D1 and D2 layer count versus required rejection ratio for the fixed channel spacing of $100 \mathrm{GHz}$.

By comparison, in their implementations of tunable TFF design Domash et al. also opted for hydrogenated amorphous silicon (a-Si:H) with the aim of exploiting its large thermo-optic coefficient for efficient thermal tuning. Their multi-cavity based design for telecom applications is formulated as follows [10]:

$$
\text { Sub. | ((HL) } \left.)^{3} \mathrm{R}_{\mathrm{S}} \mathrm{H}(\mathrm{LH})^{3} \mathrm{~L}(\mathrm{HL})^{3} \mathrm{R}_{\mathrm{S}} \mathrm{H}(\mathrm{LH})^{3}\right)^{\mathrm{R}} \mid \text { Inc. }
$$

where substrate, $\mathrm{H}$ and $\mathrm{L}$ index layer materials are identical to those used in our D1 (Table 1), whereas substrate and incidence considered are fused silica and air, respectively. Thus, these two designs are directly comparable, provided that repetition 
parameters $\mathrm{R}$ and $\mathrm{R}_{\mathrm{S}}$ yield similar transmittance shape. The main design introduced in [10] showcases the bandwidth of over $2 \mathrm{THz}\left(\mathrm{R}=1, \mathrm{R}_{\mathrm{S}}=4\right.$ and $\left.\mathrm{N}=33\right)$. Therefore, it is evident that in order to extrapolate the said design for $100 \mathrm{GHz}$ spacing in NGPON2, spacer repetition parameter RS ought to be dramatically increased. Targeting $100 \mathrm{GHz}$ spacing for IA $=35 \mathrm{~dB}$ resulted in $\mathrm{R}_{\mathrm{S}}=56$, with the design formula already reaching layer count of 137 . However, even with such significant increase of the spacer width, as shown in Fig. 4a, the said design is inappropriate for channel spacing below $200 \mathrm{GHz}$. Inherently, the design presented in [10] showcases insufficient level of sharpness for NG-PON2 application - which is resolved by increasing the number of repetitions of the main design. As evidenced by Fig.4b, by setting $R=2$ and $R_{S}=18$, the design from [10] reaches a similar $35 \mathrm{~dB}$ response to that of our D1, however with an apparent disadvantage in terms of both layer count and transmittance quality. This relates to improved flatness, higher sharpness with wider flat area, and quite importantly - significantly improved maximum isolation of our design. Maximum isolation is essential for mitigating inter-channel crosstalk, and ought to be maximized in order to assure comparable performance of a Lorentzian shape filter (TFF) to that of a flat-top super Gaussian shape (AWG). The evaluation of the impact of these performance indicators on the system level performance is discussed in the following sections.
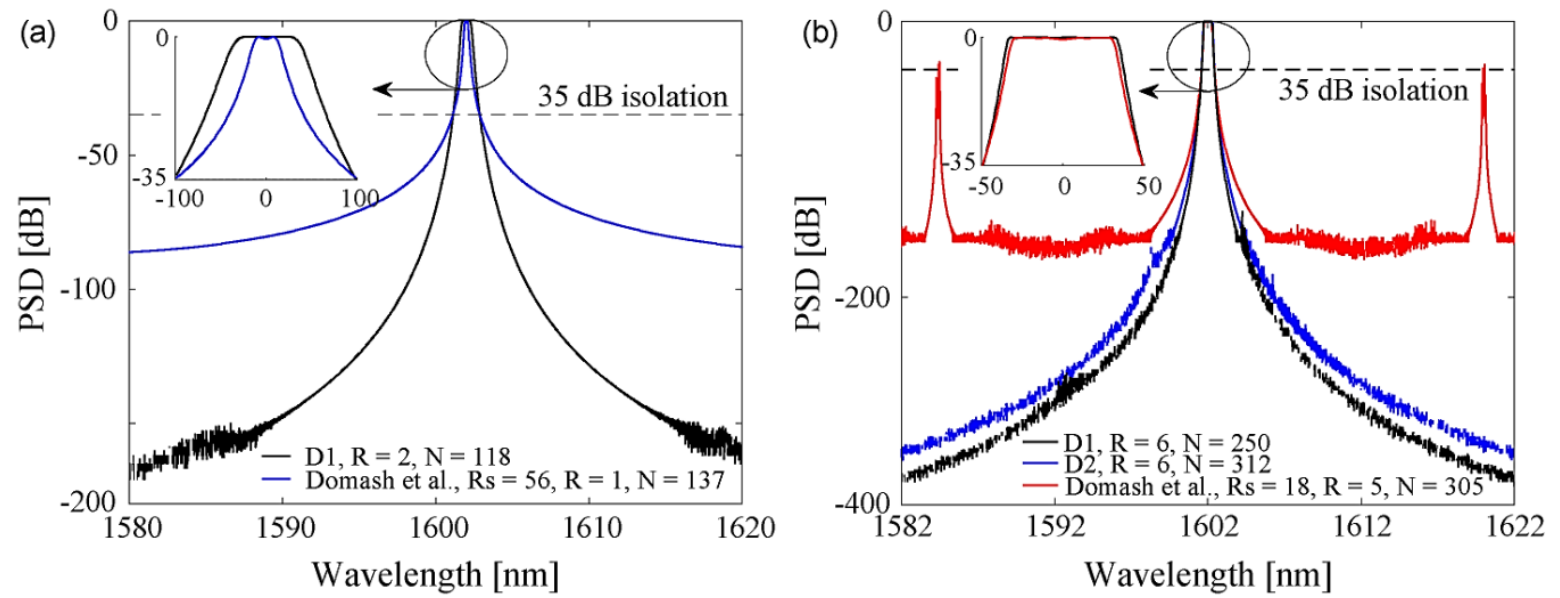

Fig. 4. a) Power spectral density (PSD) for $I_{A}=35 d B$ of $D 1$ versus that of Domash et al. design for $R_{S}=56$ and $R=1$, and $b$ ) versus same design for $R_{S}=18$ and $\mathrm{R}=5$, including $\mathrm{D} 2$. Insets show enlarged transmittance for sharpness comparison.

\section{III.TUNING STABILITY AND RANGE}

In this section, we address the tuning properties of the considered TFF designs in both angle and thermal tuning scenarios towards identifying comparative advantages. We summarize maximum ripple, bandwidth stability, as well as the required incidence angle and temperature for a range of tuning frequencies, respectively.

\section{A. Angle Tuning Scenario}

Frequency tuning shift, in-band ripple and bandwidth characterization for both polarization modes in angle tuning scenario are presented in Fig.5. Insets showcase the required incidence angle corresponding to the NG-PON2 frequency grid, along with the resulting spectral response degradation. As expected, due to the material choice and stability associated with refractive index contrast [19], D1 allows a considerably wider angle tuning range for a comparable degradation of filter response and PDL. However, both designs suffer from significant response degradation in scenarios requiring angle tuning beyond the range of $300 \mathrm{GHz}$ (NG-PON2 4-channel system). However, only a-Si-H-based D1 meets full feasibility requirements for $300 \mathrm{GHz}$ angle tuning in terms of filter transmittance, with shape and polarization related degradation within the tolerance levels. The maximum ripple in such case was $3 \mathrm{~dB}$, while filter bandwidth deviation was $3 \mathrm{GHz}$ and $10 \mathrm{GHz}$ for P- and S-polarization, respectively. The polarization separation is more severe in a-Ge-based D2, and so is the response degradation for both individual polarization states in terms of ripple and bandwidth stability. Thus, in regards to the angle tuning scenario, it is essential to stress that the design itself ought to be appropriated for angle tuning stability, even though the polarization state separation will still inherently occur [19]. In our designs, the introduction of lower $\mathrm{H}$ refractive index showcased improved stability mainly due to the fact that spacers are $\mathrm{H}$ layer based. 
(a)

Frequency tuning shift, $\Delta \mathrm{f}[\mathrm{GHz}]$
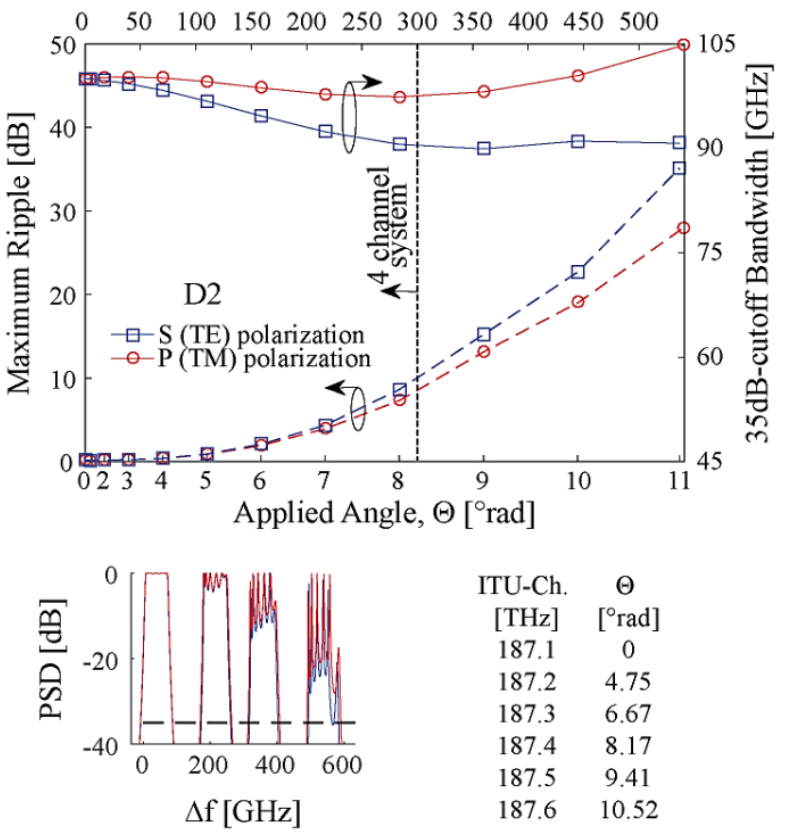

ITU-Ch. $\quad \Theta$

$[\mathrm{THz}] \quad\left[{ }^{\circ} \mathrm{rad}\right]$

$187.1 \quad 0$

$187.2 \quad 4.75$

$187.3 \quad 6.67$

$187.4 \quad 8.17$

$\begin{array}{ll}187.5 & 9.41\end{array}$

$187.6 \quad 10.52$ (b)

Frequency tuning shift, $\Delta \mathrm{f}[\mathrm{GHz}]$
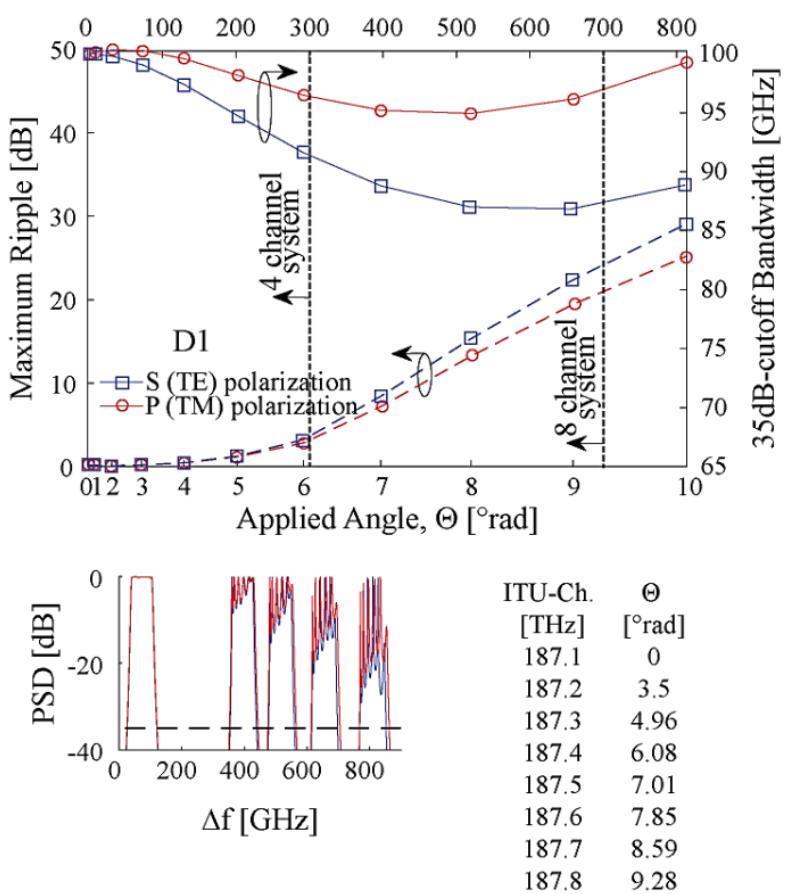

Fig. 5. a) D2 and b) D1 maximum ripple, bandwidth stability and PDL in angle tuning scenario compliant to the NG-PON2 wavelength plan in L-band. Insets show the corresponding incidence angle and resulting spectral response degradation.

Namely, L layer based spacer design is a more appropriate solution for angle tuning scenarios, as it may minimize transmittance instability and PDL. For comparison to D1, we may take a typical single-cavity TFF design relying on the L-based spacer instead [11]:

$$
\text { Inc. | }\left((\mathrm{LH})^{5} \mathrm{R}_{\mathrm{S}} \mathrm{L}(\mathrm{HL})^{4} \mathrm{H}\right)^{\mathrm{R}} \mid \mathrm{Sub} \text {. }
$$

while generalizing the formula and considering the identical materials choice to that in [11]. We calculate that, for such design, the spacer width of $\mathrm{R}_{\mathrm{S}}=36 \mathrm{QWs}$ and design repetition of $\mathrm{R}=5$, resulting in the total QW layer count of 275, would be required to meet the criteria of similar response shape to that of D1. As shown in Fig.6a, the resulting performance would constitute an improvement in shape and angular tunability compared to presented in [11]. However, from Fig. 6b, it is apparent that although the resulting design is more impervious to the effects of polarization sensitive behavior and transmittance degradation with increasing tuning angles compared to D1, the effect is still present and potentially deleterious. Therefore, it is conclusive that in some coexistence applications angle tuning TFF design based on low temperature variability materials and L-based spacer may still be the design of choice - given that PDL remains strictly within the levels of tolerance. In further comparison to D1, however, the QW layer count is increased, whereas the flatness and the maximum isolation remain inferior. Besides, angle tuned designs still exhibit the important limitation of requiring exceptional actuation precision of the order of centesimals of a degree, often accompanied by the angles beyond $20^{\circ} \mathrm{rad}$ in coexistence scenarios [12], which may introduce significant feasibility issues in the light of the current state of electrostatically driven MEMS technology [12]. Failure in meeting those requirements, on the other hand, will likely have a sizable impact in telecom applications where deviation from nominal signal frequency may result in considerable signal performance degradation, affecting the overall system feasibility. The repercussions of such filter tuning errors will be further discussed in the next section. 


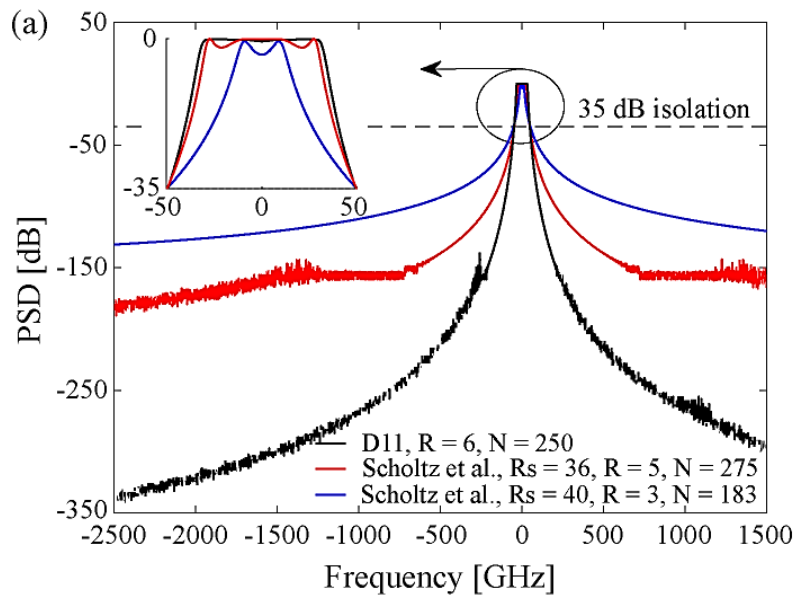

(b)

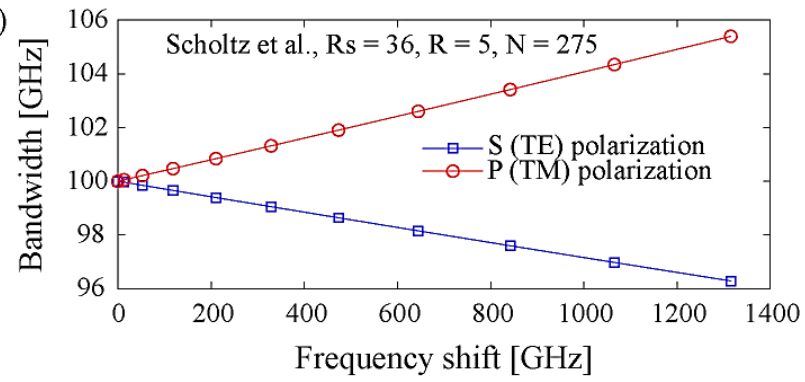

Fig. 6. a) PSD for $\mathrm{I}_{\mathrm{A}}=35 \mathrm{~dB}$ of $\mathrm{D} 1$ versus that of Scholtz et al. design considering various repetition parameters. Inset shows enlarged transmittance for sharpness and ripple comparison. b) PDL effect in Scholtz et al. design for $\mathrm{R}_{\mathrm{S}}=36$ and $\mathrm{R}=5$.

\section{B. Thermal Tuning Scenario}

The results of stability analysis in thermal tuning scenario for both designs are presented in Fig.7. Evidently, both designs exhibit higher degree of stability when compared to angular tuning, both in terms of in-band ripple, bandwidth and PDL. In fact, both designs are superior in terms of polarization loss even to the single cavity L-spacer design optimized for angle tuning (Fig.6a). It is also evident that a-Ge-based D2 allows a substantially wider thermal tuning range in comparison to a-Si-H-based D1. Namely, the average thermal tunability of $\mathrm{D} 2$ over the range of $700 \mathrm{GHz}$ is $26 \mathrm{GHz} /{ }^{\circ} \mathrm{C}$, in contrast to that of D1 equal to $10.4 \mathrm{GHz} /{ }^{\circ} \mathrm{C}$ which is very similar to what Domash et al. obtained experimentally for their own dual-cavity a-Si-H-based tunable design. Thus, by introducing a-Ge to our design, we were able to boost the thermal tunability by more than the factor of two at the cost of increased layer count. Furthermore, D2 showcased $5 \mathrm{GHz}$ of bandwidth deviation and maximum in-band ripple of only $1.6 \mathrm{~dB}$ for 8-channel system tuning range, which are both superior to the 4-channel case of D1 achieved as the best case scenario via angle tuning mechanism.

(a) Frequency tuning shift, $\Delta \mathrm{f}[\mathrm{GHz}]$
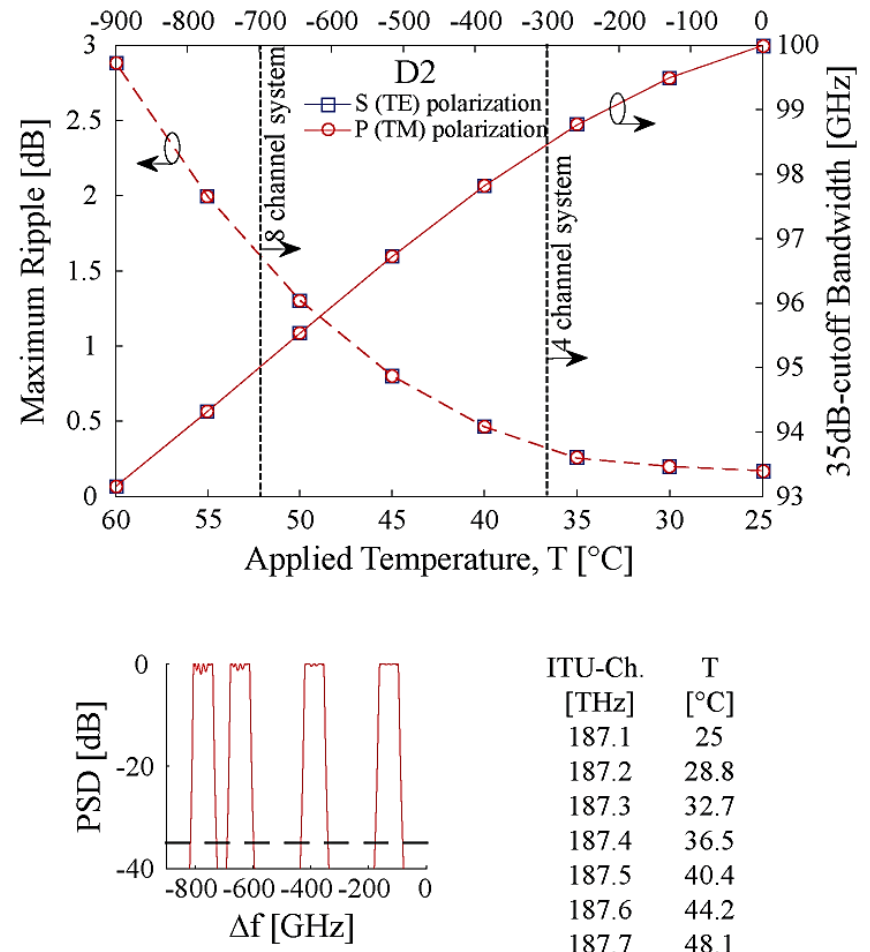

$\begin{array}{rc}\text { ITU-Ch. } & \text { T } \\ \text { [THz] } & {\left[{ }^{\circ} \mathrm{C}\right]} \\ 187.1 & 25 \\ 187.2 & 28.8 \\ 187.3 & 32.7 \\ 187.4 & 36.5 \\ 187.5 & 40.4 \\ 187.6 & 44.2 \\ 187.7 & 48.1 \\ 187.8 & 52\end{array}$

(b)
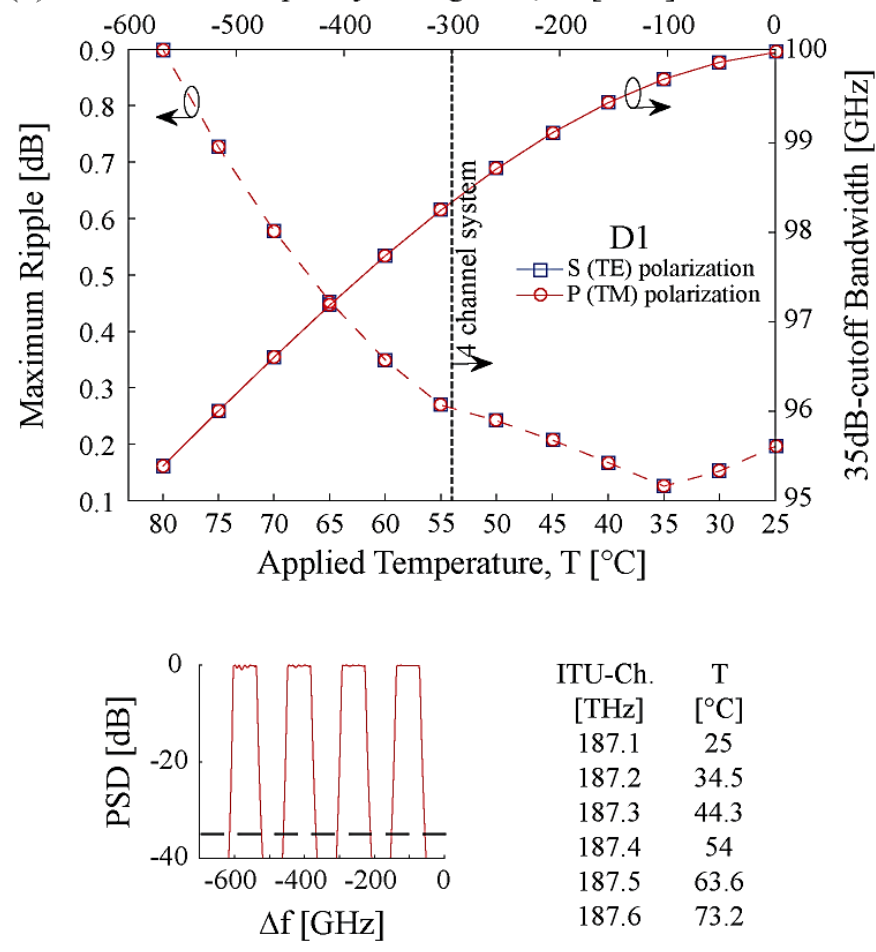

$\begin{array}{rc}\begin{array}{r}\text { ITU-Ch. } \\ \text { [THz] }\end{array} & \begin{array}{c}\mathrm{T} \\ {\left[{ }^{\circ} \mathrm{C}\right]}\end{array} \\ 187.1 & 25 \\ 187.2 & 34.5 \\ 187.3 & 44.3 \\ 187.4 & 54 \\ 187.5 & 63.6 \\ 187.6 & 73.2\end{array}$

Fig. 7. a) D2 and b) D1 maximum ripple, bandwidth stability and PDL in thermal tuning scenario compliant to the NG-PON2 wavelength plan in L-band. Insets show the corresponding required temperature and resulting spectral response degradation. 
It is important to note that, in addition to superior tuning properties, D2 showcases lower sensitivity to deviation from the normal angle of incidence stemming from potential coupling misalignment in PICs. The control of alignment precision is one of the costliest features of integrated photonics $[6,14]$, and it is thus important to consider the maximum tolerated deviation from normal angle of incidence in thermally tuned ONU components. As indicated in Fig. 8a, the frequency shift grows exponentially as the angle of incidence is increased, stressing the relevance of oblique incidence and tuning precision in this scenario. The results further indicate that, for $20 \mathrm{GHz}$ of maximum spectral excursion (MSE), D2 tolerates over $2 \square$ of $\Theta$, while D1 expectedly showcases much higher sensitivity to angle deviation. Namely, in this range of incidence angles, angle detuning sensitivity may be inherently reduced from $33 \mathrm{GHz} / \square \mathrm{rad}$ for D1 to $16 \mathrm{GHz} / \square \mathrm{rad}$ for D2. This is attributed to lower refractive index of D1 H-layers $\left(n_{H}\right)$, and the necessity to balance the temperature response of its spacer by introducing L-layers in its structure. Therefore, appropriate choice of H-layer material in our tunable TFF design is of upmost importance, not only from the perspective of thermo-optical coefficient, but in particular since $n_{H}$ plays a dominant role in angular sensitivity. Conversely, L-layer refractive index $n_{L}$ has a low impact on $\Theta$ sensitivity in H-dominated spacer design, as demonstrated by the frequency tuning contours presented in Fig. 8b. In effect, for $n_{L}$ ranging between 1.5 and 2.5, the angular stability for a constant frequency shift remains within $0.2^{\circ}$. Thus, L-layer material choice ought to be primarily motivated by achieving proper transmittance characteristics, rather than mitigating the angular misalignment sensitivity.

(a)

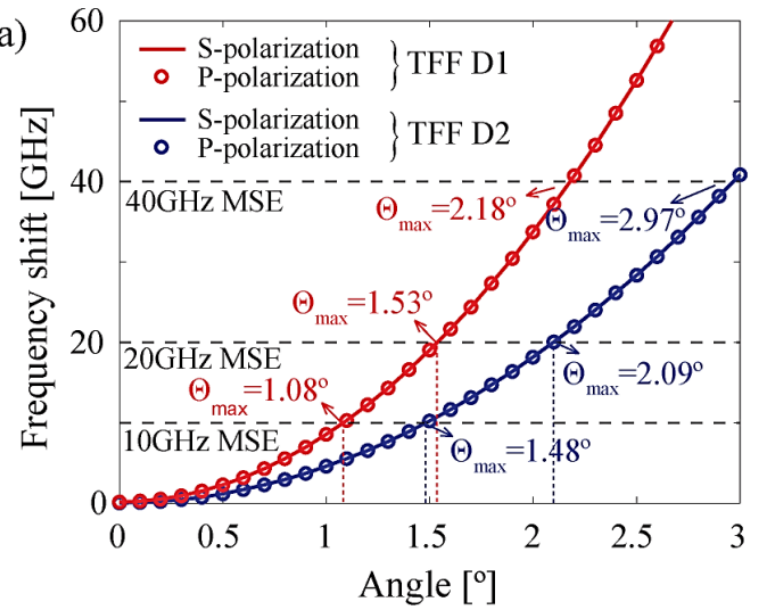

(b)

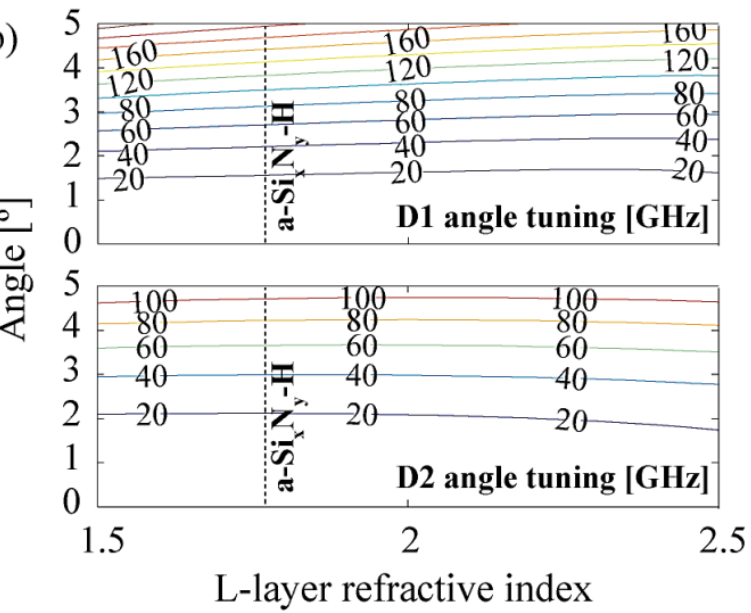

Fig. 8. a) D1 and D2 sensitivity to oblique incidence, highlighting their level of compliance to the MSE requirements. b) D1 (upper) and D2 (lower) angle tuning sensitivity contours versus incidence angle $\Theta$ and L-layer refractive index $n_{L}$.

\section{IV.CROSSTALK TOLERANCE}

In this section we evaluate the repercussions of TFF design on inter-channel crosstalk isolation along with the resulting signal penalty and tuning error tolerance in NG-PON2 scenario.

System set-up used for performance assessment is presented in Fig.9a. The simulated scenario entails both 8-channel NGPON2 $(8 \times 10 \mathrm{~Gb} / \mathrm{s})$ and 4-channel NG-PON2 $(4 \times 25 \mathrm{~Gb} / \mathrm{s})$ variants, with $100 \mathrm{GHz}$ channel spacing in both cases (the resulting spectral efficiencies being $0.1 \mathrm{bit} / \mathrm{s} / \mathrm{Hz}$ and $0.25 \mathrm{bit} / \mathrm{s} / \mathrm{Hz}$, respectively). The simulation was carried out using previously validated models [5]. We considered remote node (RN) architecture based on both flat-top AWG wavelength demultiplexer (WD), modeled as the $2^{\text {nd }}$ order super-Gaussian (SG) filter, as well as on passive splitter (PS) paired with TFFs for channel selection at the ONU. The transmission line is modeled as a $20 \mathrm{~km}$ long standard single mode fiber (SSMF) [30], considering the effective area of $80 \mu \mathrm{m}^{2}$, dispersion slope of $0.06 \mathrm{ps} /\left(\mathrm{nm}^{2} \cdot \mathrm{km}\right)$, dispersion parameter of $16 \mathrm{ps} /(\mathrm{nm} \cdot \mathrm{km})$, and nonlinearity coefficient of $1.62(\mathrm{~W} \cdot \mathrm{km})^{-1}$. Decorrelated DeBruijn binary sequences of $2^{12}$ bits were used for the encoding of different WDM channels, towards rigorous assessment of multichannel nonlinear transmission system performance [31]. Signal performance was evaluated by Q-factor calculation based on Gaussian approach, elected over other similar models (e.g. the exact method, saddle-point approximation) due to comparable accuracy and considerably lower computational latency [8,9]. Signal sensitivity was assessed at $\mathrm{BER}=1 \cdot 10^{-4}$, which corresponds to the conventional hard decision FEC with $12 \%$ overhead [32]. Electrical filter of the PIN receiver was assumed to be a 4th order Bessel filter, with optimized $3 \mathrm{~dB}$ bandwidth of $0.7 \mathrm{R}_{b}, \mathrm{R}_{\mathrm{b}}$ being the signal data rate. The launch power per-channel considered was $10 \mathrm{dBm}$, compliant with the NG-PON2 class E2 [1], whereas the signal extinction ratio (ER) was set to $8.2 \mathrm{~dB}$ [1]. Transmitter relative intensity noise (RIN) considered in this work is $-160 \mathrm{~dB} / \mathrm{Hz}$ [5]. 
(a)

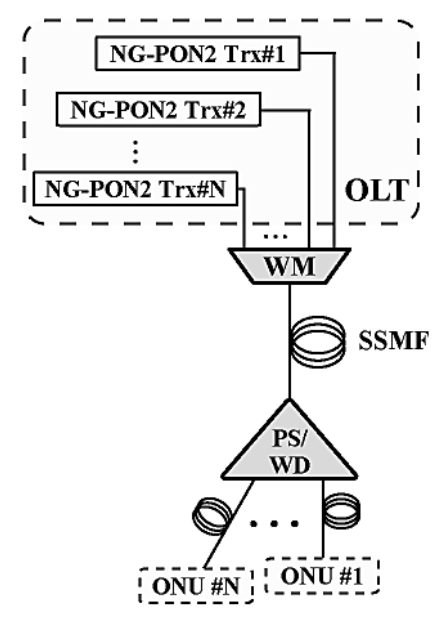

(b)

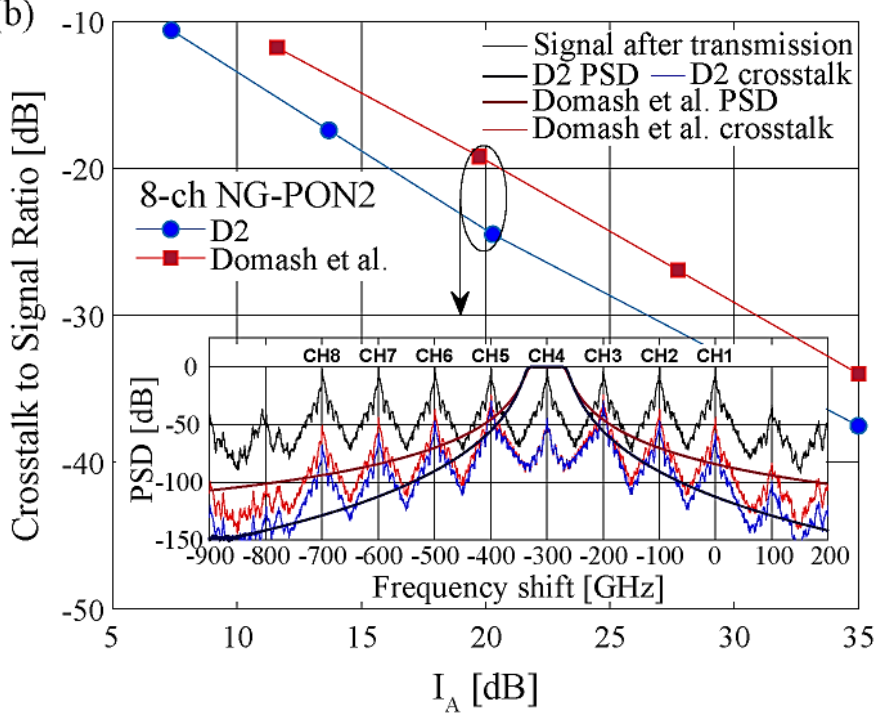

Fig. 9. a) Simulation setup for system performance evaluation in NG-PON2 scenario. b) Crosstalk to signal ratio comparison between D2 and Domash et al. for zero detuning, considering a range of $\mathrm{I}_{\mathrm{A}}$. Inset shows the PSD of multiplexed channels after fiber transmission, crosstalk PSD and filter transmittance for both TFF designs.

In order to comparatively assess the isolation properties of candidate filters, we first considered the crosstalk to signal ratio in the 8-channel NG-PON2 scenario when no filter detuning from the signal frequency is applied. The results presented in Fig.9b indicate an improvement of D2 inter-channel crosstalk isolation of over $5 \mathrm{~dB}$ compared to the design of Domash et al. [10]. The inset of Fig.9b shows the WDM signal PSD after fiber transmission, along with the crosstalk PSD upon optical filtering, for both TFF designs.

We next assessed the filter crosstalk isolation in the scenario of TFF central frequency detuning and its misalignment from the transmitter nominal frequency, potentially caused by thermal control instability. Fig.10a shows the crosstalk to signal ratio for a range of filter detuning frequencies, considering several filter designs and $I_{A}=20 \mathrm{~dB}$. The insets show the signal sensitivity degradation for increased OFIL spectral detuning, as well as the eye opening degradation with increasing crosstalk level. Clearly, for the same level of inter-channel crosstalk, the tuning error tolerance highly depends on the filter design. In direct comparison to the design in [10], the potentially lower tuning control stability of D2 stemming from the discussed doubled thermal tunability coefficient is evidentially offset by much improved inter-channel crosstalk isolation.
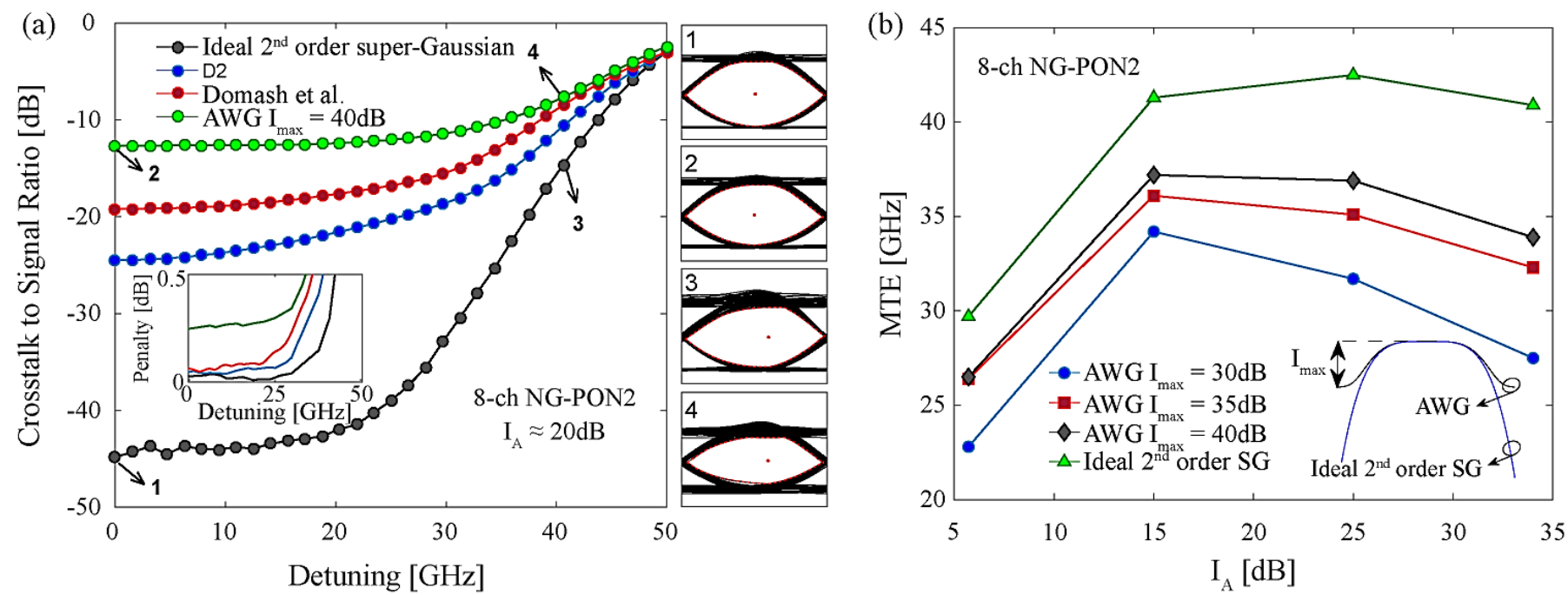

Fig. 10. a) Crosstalk to signal ratio versus OFIL detuning from the nominal signal frequency for several TFF and AWG designs. Insets show the respective sensitivity and eye diagram degradation. b) AWG MTE for various levels of $\mathrm{I}_{\max }$ in 8-channel NG-PON2 system.

In the asessment of the maximum tuning error (MTE), we consider the maximum tolerated TFF detuning for $0.5 \mathrm{~dB}$ of power penalty compared to zero detuning, in all considered cases. The OFIL detuning was applied to the channel under analysis only, and thus represents the absolute value of tolerated MTE. 
The lack of transmittance skirt in the SG response of an ideal flat-top AWG model expectedly yields superior crosstalk isolation compared to the Lorentzian shape of a TFF design. However, results for the 8-channel NG-PON2 scenario presented in Fig.10b showcase high sensitivity of AWG MTE to the value of maximum adjacent channel isolation of the filter response $\mathrm{I}_{\max }$, over a wide range of $\mathrm{I}_{\mathrm{A}}$. Consequently, compared to that of the ideal $2^{\text {nd }}$ order $\mathrm{SG}$, a more realistic flat-top AWG filter response with maximum isolation of less than $40 \mathrm{~dB}$ yields considerably lower detuning tolerances.
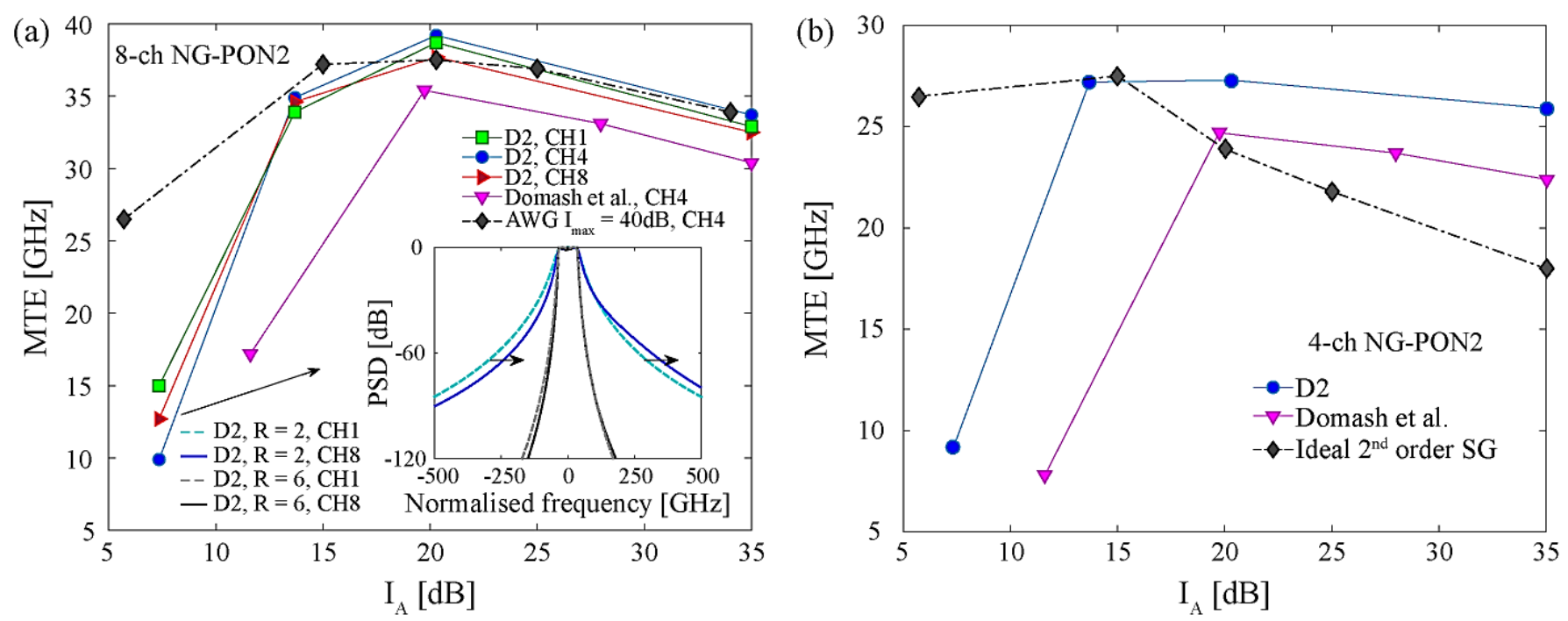

Fig. 11. a) MTE for D2 and Domash et al. TFF designs in comparison to reference AWG with $\mathrm{I}_{\max }=40 \mathrm{~dB}$, for $8 \times 10 \mathrm{~Gb} / \mathrm{s}$ system. Inset demonstrates the effect of skirt skewing at low values of $\mathrm{I}_{\mathrm{A}}$. b) MTE for D2 and Domash et al. TFF designs in comparison to reference ideal $2^{\text {nd }}$ order SG filter, for 4x25 Gb/s system.

Fig.11a shows the comparative MTEs for D2 and Domash et al. $\left(\mathrm{R}_{\mathrm{S}}=18\right)$ TFF designs, as well as the reference case of filtering by AWG with $\mathrm{I}_{\max }=40 \mathrm{~dB}$. The results indicate that, in 8-channel NG-PON2 scenario, TFF D2 outperforms that of [10] for the full range of $\mathrm{I}_{\mathrm{A}}$, whereas it outperforms AWG for $\mathrm{I}_{\mathrm{A}}>15 \mathrm{~dB}$ and $\mathrm{I}_{\max }<40 \mathrm{~dB}$. Evidently, MTE has an optimal value as the result of a trade off between increasing level of inter-channel crosstalk for lower $\mathrm{I}_{\mathrm{A}}$ values, and increased inter-symbol interference (ISI) imposed by detuned filter response of higher sharpness (higher $\mathrm{I}_{\mathrm{A}}$ values). A substantial inconsistency in $\mathrm{D} 2$ performance across different channels in thermal tuning scenario occurs solely for $\mathrm{I}_{\mathrm{A}}$ values below $10 \mathrm{~dB}$, where performance is dominatly affected by the inter-channel crosstalk. The latter occurs due to the effect of the transmittance skirt skewing, which is significantly more pronounced for low values of $\mathrm{I}_{\mathrm{A}}$, as shown in the inset of Fig.11a. Nevertheless, the optimal performance is found around $\mathrm{I}_{\mathrm{A}}=20 \mathrm{~dB}$, which corresponds to the main period repetition of $\mathrm{R}=4$ and results in QW layer count of $\mathrm{N}=222$. These specifications may thus be considered optimal for improved MTE performance compared to traditional thermal filtering solutions based on state of the art TFFs, and flat-top AWG designs with $\mathrm{I}_{\max }<40 \mathrm{~dB}$.

As mentioned, our TFF design methodology entailed calculation of the matching layer composition to yield optimal performance for the highest frequency channel in all considered cases of $\mathrm{D} 1$ and $\mathrm{D} 2$, as to assess the tuning performance and its effect on response degradation more rigorously. However, it should be noted that the penalty resulting from transmittance degradation with increasing tuning range may be offset by optimising the matching layer stack design to the frequency of the central channel instead, particularly for channel count beyond 8 .

Finally, as per-channel capacity is increased, so is the advantage of utilizing TFFs as opposed to AWG, as can be seen from Fig. $11 \mathrm{~b}$. For the $4 \times 25 \mathrm{~Gb} / \mathrm{s}$ flavor of NG-PON2 [3], we found that $\mathrm{D} 2$ provides improved detuning tolerance for a full $\mathrm{I}_{\mathrm{A}}$ range when compared to the design of Domash et al., and for $\mathrm{I}_{\mathrm{A}}>15 \mathrm{~dB}$ compared to the ideal $2^{\text {nd }}$ order $\mathrm{SG}$ filter. Thus, $25 \mathrm{~Gb} / \mathrm{s}$ perchannel operation scenario is where the introduction of appropriate TFF design is expected to yield a considerable advantage in terms of crosstalk isolation and the penalty of tuning control instability. The following section outlines the advantages of our design stemming from improved latency and power consumption in mentioned scenario.

\section{TUNING TIME AND POWER CONSUMPTION}

As evident from Table 1, the choice of materials for thermally tuned TFF design yields specific tradeoffs between the optical performance, tuning temperature range and layer count. However, thermal conductivity of elected materials is another crucial factor to be considered towards reduced power consumption. Tuning time and power consumption investigation was carried out using ANSYS ${ }^{\circledR}$ software, relying on finite element method with predefined heat transfer mechanisms, initial and boundary conditions. Hence, each layer is modeled as a $500 \times 500 \mu \mathrm{m}^{2}$ area with variable thickness, following the TFF layout with respect to TEC established in Fig. 1. The simulation allows intra and inter-layer heat transfer by conduction, however ignoring negligible convection and radiation mechanisms. Conduction heat transfer within a $50 \mu \mathrm{m}$ thick $\mathrm{Si}$ substrate and the film-substrate interface are also considered, along with the insulation effect provided by the Epoxy, as shown in Fig.12a. 

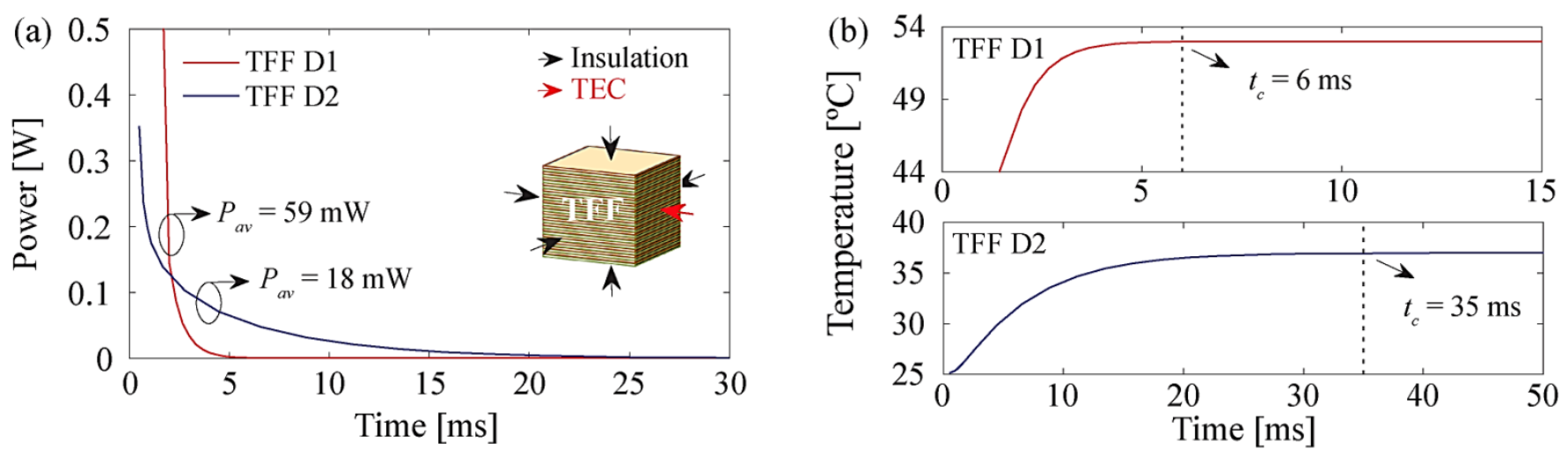

Fig. 12. a) TEC power consumption for D1 and D2. Inset shows TFF geometry relative to TEC. b) D1 (upper) and D2 (lower) tuning times, needed for stabilization of temperature required for the full tuning range over $300 \mathrm{GHz}, \Delta T$.

Towards a fair comparison, tuning time and power consumption analysis was performed within the frequency range that is feasible for both TFF designs, i.e. the 4-channel NG-PON2 scenario. Assuming a step function for TEC thermal excitation, higher heat load required for D1 tuning leads to reduced tuning time compared to D2, as shown in Fig.12b. Fig.12a shows that the latter comes at the cost of significantly increased average power consumption, as D2 allows power efficiency increase by factor 3.28 while still well within the standardized class 3 tuning time requirements [9].

Thus, described TFF design methodology, involving a careful selection of H and L materials, enables controlled tradeoff between filter complexity, tuning time and power consumption towards their low-cost application in PON. The obtained results for power consumption were compared to those proposed by Yang et al [13] for thermally tuned AWG, within the $300 \mathrm{GHz}$ spectral separation. Indeed, our filtering approach demonstrated substantially higher energy efficiency, requiring driving power of $18 \mathrm{~mW}$, in contrast to $500 \mathrm{~mW}$ reported for AWG [13]. Furthermore, the obtained tuning time falls within with Class 2 devices (10 $\mu$ s to $25 \mathrm{~ms}$ ) compliant with channel-based protection mechanisms $(50 \mathrm{~ms})$. However, D2 results still indicate below Class 1 for tunable devices, necessary to enable dynamic wavelength and bandwidth allocation [16]. However, we also remark that, as D1 results show, power efficiency may be traded off for lower latency by means of proper selection of H-layer material, and still remain superior to that reported in [13].

\section{VI.SUMMARY}

We investigate the feasibility of thermally tuned TFFs for NG-PON2 applications in both $8 \times 10 \mathrm{~Gb} / \mathrm{s}$ and $4 \times 25 \mathrm{~Gb} / \mathrm{s}$ flavors. We present unique TFF designs for $100 \mathrm{GHz}$ channel spacing, relying on contrasting materials relevant to their optical performance and tunability, both showcasing beyond state of the art in-band flatness and excellent sharpness. We identify advantages over mechanically tuned TFF design approach in PIC scenario, such as relaxed coupling alignment control requirements, reduced physical footprint and complexity. Through detailed comparative analysis, we find that when optimized towards those scenario strengths, our thermally tuned TFF design offers improvements in aspects of polarization stability, crosstalk tolerance, and overall system feasibility when compared to typical angle tuning optimized designs. Furthermore, we identify relevant component and system level tradeoffs to be exploited towards considerably reduced power consumption and increased tuning stability compared to the current state of the art TFFs and AWGs with limited maximum spectral isolation.

\section{ACKNOWLEDGEMENTS}

This work was partially funded by the European Union's Horizon 2020 for research, technological development, and demonstration under grant agreement no. 688941 (FUTEBOL). Additional support was provided by the European Regional Development Fund (FEDER), through the Regional Operational Program of Centre (CENTRO 2020) of the Portugal 2020 framework [Project HeatIT with Nr. 017942 (CENTRO-01-0247-FEDER-017942)]. The FCT under the scholarship SFRH/BPD/119188/2016 is also acknowledged.

\section{REFERENCES}

[1] ITU-T G.989.2 Amendment 2, "40-Gigabit-capable passive optical networks 2 (NG-PON2): Physical media dependent (PMD) layer specification", (2017).

[2] D. Nesset, "PON roadmap [invited]," in IEEE/OSA Journal of Optical Communications and Networking, vol. 9, no. 1, pp. A71-A76, Jan. 2017.

[3] V. Houtsma, D. van Veen, S. Porto, N. Basavanhally, C. Bolle, and H. Schmuck, "Investigation of 100G (4x25G) NG-PON2 Upgrade using a Burst Mode Laser based on a Multi-Electrode Laser to enable $100 \mathrm{GHz}$ Wavelength Grid," in Optical Fiber Communication Conference, OSA Technical Digest (online) (Optical Society of America, 2018), paper M1B.3.

[4] Ovum, "Broadband access equipment forecast (PON, xDSL, CMTS): 2015-21," TE0006-001186, Feb. 2016 [Online].

[5] . Z. Vujicic, et al., "Considerations on Performance, Cost and Power Consumption of Candidate 100G EPON Architectures [Invited]," Proc. ICTON, Trento, Italy (2016).

[6] E. R. H. Fuchs, E. J. Bruce, R. J. Ram, and R. E. Kirchain, "Process-based cost modeling of photonics manufacture: The cost competitiveness of monolithic integration of a 1550-nm DFB laser and an electroabsorptive modulator on an InP platform," J. Light. Technol., vol. 24, no. 8, pp. 3175-3186, Aug. 2006.

[7] P. Groumas et al., "Novel photonic integration platform based on electro-optic polymers," 2012 38th European Conference and Exhibition on Optical Communications, Amsterdam, 2012, pp. 1-3. 
[8] Zhang, Z.; Felipe, D.; Brinker, W.; Kleinert, M.; Maese-Novo, A.; Moehrle, M.; Zawadzki, C.; Keil, N. C/L-Band Colorless ONU Based on Polymer Bidirectional Optical Subassembly. IEEE J. Lightwave Technol. 2015, 33, 1230-1234.

[9] N. Uehara et al., "Advanced Band Separation Thin-Film Filters for Coexistence-Type Colorless WDM-PON," Proc. OFC, p. OWI4 (2008).

[10] L. Domash, et al., "Tunable and switchable multiple-cavity thin film filters," in Jour. of Light. Tech., vol. 22, no. 1, pp. 126-135 (2004).

[11] L. Scholtz, D. Korcek, L. Ladányi and J. Müllerová, "Tunable thin film filters for the next generation PON stage 2 (NG-PON2)," 2014 ELEKTRO, Rajecke Teplice, 2014, pp. 98-102.

[12] O. Solgaard, A. A. Godil, R. T. Howe, L. P. Lee, Y. A. Peter and H. Zappe, "Optical MEMS: From Micromirrors to Complex Systems," in Journal of Microelectromechanical Systems, vol. 23, no. 3, pp. 517-538, June 2014.

[13] Andreas C. Fischer, Fredrik Forsberg, Martin Lapisa, Simon J. Bleiker, Göran Stemme, Niclas Roxhed \& Frank Niklaus, "Integrating MEMS and ICs", Microsystems \& Nanoengineering volume 1, Article number: 15005 (2015).

[14] Q. X. Zhang et al., "A Silicon Platform with MEMS Active Alignment Function and Its Potential Application in Si-Photonics Packaging," in IEEE Journal of Selected Topics in Quantum Electronics, vol. 16, no. 1, pp. 267-275, Jan.-feb. 2010.

[15] K. Yu et al., "Design of non-polarization angle-tuned narrowband thin film filter", Jour. Light Elect. Opt., vol. 125, pp. 397-400 (2014).

[16] Y. Yang et al., "Thermo-Optically Tunable Silicon AWG with Above $600 \mathrm{GHz}$ Channel Tunability," in IEEE Photonics Technology Letters, vol. 27, no. 22, pp. 2351-2354, Nov.15, 152015.

[17] Y. Luo et al., "Physical layer aspects of NG-PON2 standards-Part 2: System design and technology feasibility [Invited]," in IEEE/OSA Journal of Optical Communications and Networking, vol. 8, no. 1, pp. 43-52, January 12016.

[18] Fredrik Ahlbom. (2016). Analysis of the Performance of Different DWDM Filter Technologies for Mobile Fronthaul Applications (Master dissertation), Kth Royal Institute of Technology of Information and Communication Technology, Stockholm, Sweden.

[19] A. Macleod, "Thin-Film Optical Filters" (Taylor \& Francis), Chap. 17 (2010).

[20] Antonio R. Zanatta and Ivan B. Gallo, "The Thermo Optic Coefficient of Amorphous SiN Films in the Near-Infrared and Visible Regions and Its Experimental Determination”, Applied Physics Express 6 (2013) 042402.

[21] Kevin Luke, Yoshitomo Okawachi, Michael R. E. Lamont, Alexander L. Gaeta, and Michal Lipson, "Broadband mid-infrared frequency comb generation in a Si3N4 microresonator," Opt. Lett. 40, 4823-4826 (2015).

[22] H. H. Li, "Refractive index of silicon and germanium and its wavelength and temperature derivatives", Journal of Physical and Chemical Reference Data 9, $561(1980)$.

[23] J. E. Cunningham, et al., "Highly-efficient thermally-tuned resonant optical filters," Opt. Express 18, 19055-19063 (2010).

[24] T. Z. Zhan et al., "Thermal conductivity of sputtered amorphous Ge films", AIP Adv. 4, 027126 (2014).

[25] D. G. Cahill et al., "Thermal conductivity of a-Si:H thin films", Phys. Rev. B 50, 6077 (1994).

[26] Available at: www.norlandprod.com/adhesives/noa\%2065.html.

[27] T.W.Tong, “Thermal Conductivity,” In Proc. Con. Therm. Cond., 22, pp 674 (1994).

[28] H. Jianqiang, et al., "Thermal conductivity of PECVD Si-rich silicon nitride films measured with a SiO2/SixNy biomaterial microbridge test structure", J. Semic. 35 (2014).

[29] E. Hossain et al., "5G Cellular: Key Enabling Technologies and Research Challenges”, IEEE Inst. and Meas. Mag., vol. 18, no. 3 pp. 11-21 (2015).

[30] E. Iannone, et al., Nonlinear optical communication networks, John Wiley \& Sons, ch. 2 and 3 (1998).

[31] A. B. Carlson, et al., Communication systems - an introduction to signals and noise in electrical communication, McGraw-Hill, New York, ed. 4, ch. 1 and 4 (1995).

[32] Dave Hood, Elmar Trojer (2012), Gigabit-capable Passive Optical Networks, Appendix I, Wiley. 